\author{
Corpus \\ CORPUS \\ 13 | 2014 \\ Eléments initiaux dans la phrase : approches inter- \\ genres et inter-langues
}

\title{
Les éléments attributifs en position initiale en allemand et leurs équivalents en français - étude de corpus contrastive
}

Séverine Adam et Cécile Delettres

\section{OpenEdition}

Journals

Édition électronique

URL : http://journals.openedition.org/corpus/2476

DOI : $10.4000 /$ corpus. 2476

ISSN : $1765-3126$

Éditeur

Bases; corpus et langage - UMR 6039

Édition imprimée

Date de publication : 1 décembre 2014

Pagination : 165-190

ISSN : 1638-9808

Référence électronique

Séverine Adam et Cécile Delettres, "Les éléments attributifs en position initiale en allemand et leurs équivalents en français - étude de corpus contrastive », Corpus [En ligne], 13 | 2014, mis en ligne le 01 mai 2015, consulté le 08 septembre 2020. URL : http://journals.openedition.org/corpus/2476 ; DOI : https://doi.org/10.4000/corpus.2476

(c) Tous droits réservés 


\title{
Les éléments attributifs en position initiale en allemand et leurs équivalents en français - étude de corpus contrastive
}

\author{
Séverine ADAM \\ Cécile DELETTRES \\ CeLiSo. Paris-Sorbonne (EA 7332)
}

Cette étude se situe dans le prolongement d'un travail contrastif multilingue effectué dans le cadre du projet Eurogr@mm ${ }^{1}$, dont le dernier volet a été consacré à l'étude contrastive de l'occupation de la première position dans les langues des pays partenaires - allemand, français, hongrois, italien, norvégien, polonais. Ces travaux s'appuient sur des corpus annotés (avec Tree Tagger) élaborés spécialement pour ce projet par les informaticiens de l'IDS et rassemblant l'ensemble des articles Wikipedia (état janvier 2011) pour chacune des langues concernées. Ces corpus ont été ajoutés à la collection de corpus DeReKo ${ }^{2}$ et sont mis à la disposition des membres du projet via la plateforme Cosmas. Les recherches menées dans ce cadre le sont donc sur des corpus 'comparables' tant au niveau de la taille que du contenu et $\mathrm{du} / \mathrm{des}$ genre(s) textuel(s) :

Corpus Wikipedia allemand (C W All) :

2.137.295 textes / 551.090.404 mots

Corpus Wikipedia français (C W Fr) :

2.309.599 textes / 526.944.992 mots

L'observation approfondie de ces corpus nous a permis de confirmer et d'étayer empiriquement un certain nombre de

${ }^{1}$ Projet européen coordonné par 1'Institut für Deutsche Sprache (IDS) de Mannheim et réunissant - outre les chercheurs de l'IDS - des universités de cinq pays européens (Budapest, Gênes, Oslo, Naples, Palerme, Paris, Salerne, Szeged, Wroclaw). Un des axes principaux du projet, mené sur la période 2007-2012, a été l'élaboration d'une grammaire interactive en ligne avec approche contrastive. http://hypermedia.ids-mannheim.de/call/ public/gruwi.ansicht? v_typ $=$ o.

2 Deutsches Referenzkorpus (pour davantage d'information on se reportera aux publications de Kupietz et al.).

Corpus $n^{\circ} 13$ « Eléments initiaux dans la phrase : approches inter-genres et inter-langues » (2014), 165-190 


\section{S. AdAm, C. Delettres}

présomptions concernant les caractéristiques morpho-syntaxiques et sémantiques des unités apparaissant en tête d'énoncé, et de mettre en lumière certains traits communs au français et à l'allemand. Ainsi est-il par exemple apparu que, dans les deux langues, les éléments initiaux sont, exception faite des connecteurs, généralement référentiels.

Cependant, ces mêmes recherches ont également fait apparaître des différences fondamentales entre les deux langues : les études menées sur le corpus allemand ont par exemple - et c'est le cas sur lequel le présent article entend s'attarder - mis en lumière la très grande fréquence, en position initiale, d'unités nominales non référentielles remplissant la fonction syntaxique d'attribut du sujet, comme dans les exemples (1) et (2) :

(1) Ziel der Forschung sind elektrische Kiemen. [But de la recherche sont des branchies électriques.] $]^{3}$

(2) Ein Problem sind zurzeit die Bildschirme. [Un problème sont actuellement les écrans.]

Dans cette même fonction, à cette même position et avec une fréquence tout aussi significative se rencontrent également toute une série d'unités adjectivales, dans des énoncés tels que ceux proposés sous (3) et (4) :

(3) Wichtig ist auch, dass die Handschuhe auf keinen Fall zu eng sitzen, weil das sonst die Durchblutung stören würde.

[Important est aussi que les gants ne soient en aucun cas trop serrés car cela pourrait perturber la circulation sanguine.]

(4) Auffällig ist auch die hohe Anzahl an Asthmatikern und chronisch Kranken unter den Radsportlern.

[Frappant est également le grand nombre d'asthmatiques et de malades chroniques parmi les cyclistes.]

Le constat de la fréquence des énoncés de ce type soulève un certain nombre de questions tant du point de vue monolinguistique que du point de vue contrastif. D'un point de vue mono-

\footnotetext{
${ }^{3}$ Nous donnons ici entre crochets une traduction littérale des exemples allemands, agrammaticale en français mais qui permet de mettre en évidence les différences structurelles sur lesquelles se concentre notre étude. Nous reviendrons dans la deuxième partie de cette contribution sur les possibles équivalents français à ces structures.
} 
linguistique, la fréquence des structures de ce type ne va pas forcément de soi dans la mesure où il s'agit a priori de structures marquées - et ce à au moins deux titres, puisqu'elles bousculent au moins deux tendances générales (non spécifiques à l'allemand) présidant à la linéarisation des énoncés. La première de ces tendances est d'ordre sémantique : dans un énoncé, les éléments référentiels ont tendance à apparaître avant les éléments prédicatifs, dont ils constituent le support thématique. La deuxième tendance est d'ordre syntaxique : prototypiquement, le sujet syntaxique se trouve dans la périphérie gauche de l'énoncé et précède les groupes en fonction d'objet ou d'attribut. Or les énoncés qui nous intéressent vont à l'encontre de cette double tendance. Un troisième critère, propre à l'allemand cette fois, permet de considérer ces structures comme marquées : elles sont en effet en décalage par rapport à l'organisation syntaxique de l'énoncé de base en allemand; nous y reviendrons dans le premier point de cette contribution. Pour le moment, on ne peut que constater le 'paradoxe' existant a priori entre la fréquence d'emploi de ces structures et leur caractère syntaxiquement et sémantiquement marqué - la question se pose de savoir comment cela se justifie. Nous tâcherons de montrer que l'explication est à rechercher du côté des fonctions textuelles que ces structures remplissent.

Les configurations de ce type sont également intéressantes du point de vue contrastif dans la mesure où elles sont a priori sans équivalent direct/littéral en français standard contemporain - où tout complément ${ }^{4}$ en position préverbale est a priori en fonction de sujet ${ }^{5}$. Cela ne signifie pas pour autant qu'il ne soit pas possible de trouver en français des équivalents fonctionnels à ces tournures. Car les fonctions textuelles remplies par les attributs allemands initiaux sont des fonctions communes aux deux langues - c'est la première chose qu'il s'agira de mettre

\footnotetext{
${ }^{4}$ La notion de complément est purement syntaxique, elle désigne les groupes syntaxiquement nécessaires à la complémentation du verbe (sujet, objet, complément d'attribution, etc.) par opposition aux suppléments (circonstants, etc.). Pour plus de détails voir : http://hypermedia.ids-mannheim. $\mathrm{de} /$ call/public/termwb.ansicht?v_app=p\&v_id=89.

${ }^{5}$ Les quelques (rares) exceptions à ce principe seront commentées plus bas.
} 


\section{S. ADAM, C. Delettres}

en évidence dans cette contribution. Mais il s'agira d'autre part de montrer que, pour des fonctions similaires, les deux langues développent des stratégies différentes et de mettre en lumière les facteurs syntaxiques, sémantiques et pragmatiques susceptibles d'expliquer ces différences.

La première partie de cette contribution porte sur le corpus allemand et est consacrée à la description des éléments initiaux (EI) apparaissant en fonction d'attribut. Sont ensuite passées en revue les fonctions (textuelles) de ces structures marquées : l'analyse de séquences textuelles tirées du corpus permet de mettre en lumière le rôle joué par les énoncés concernés dans la gestion de la structure informationnelle.

La seconde partie de cette contribution se penche sur les équivalents fonctionnels français aux structures allemandes étudiées en amont et propose un inventaire, parallèle à celui entrepris pour l'allemand, des tournures françaises que l'on peut considérer comme (textuellement) équivalentes des structures attributives initiales allemandes, ainsi que la description de leurs spécificités formelles et fonctionnelles.

\section{Les éléments attributifs en position initiale en allemand}

\subsection{Principes d'organisation de l'énoncé allemand}

La possibilité de trouver en allemand, en position initiale, des éléments en fonction d'attribut du sujet tient à la flexibilité syntaxique de l'énoncé assertif allemand, dont les constituants sont globalement plus mobiles que les constituants du français. Ceci s'explique d'une part par l'existence d'un système de cas auquel est largement dévolu le marquage fonctionnel des constituants, d'autre part par les principes généraux d'organisation de l'énoncé - pince verbale, régressivité, champs positionnels. La notion de 'pince' verbale (Verbalklammer) décrit la structure discontinue du groupe verbal allemand (au sens large), et renvoie à la fonction de délimitation remplie par certains éléments prédicatifs. La pince verbale est constituée de deux bornes (borne gauche, borne droite) qui délimitent trois champs positionnels principaux - le champ I (Vorfeld), qui ne peut prototypiquement accueillir qu'un constituant, le champ II (ou champ médian, Mittelfeld), qui s'organise selon un certain nombre de principes 
Les EI attributifs allemands et leurs équivalents en français

syntaxiques, sémantiques et pragmatiques ${ }^{6}$, et le champ III (ou après-dernière position, Nachfeld), dont l'occupation est facultative et répond à des objectifs textuels spécifiques.

La borne gauche est toujours réalisée par la partie conjuguée du verbe, qui suit obligatoirement et immuablement le premier constituant de l'énoncé assertif; la borne droite est réalisée soit sous la forme d'un élément verbal (participe, infinitif, préverbe séparable), soit par un élément qui entretient un rapport syntaxico-sémantique privilégié avec le verbe - il peut par exemple s'agir d'un groupe nominal ou prépositionnel faisant partie d'une locution verbale (du type zur Verfügugng stellen - mettre à disposition) ou d'une unité nominale ou adjectivale en fonction d'attribut (Pilot werden - devenir pilote; witzig sein - être drôle). Dans le tableau ci-dessous sont proposés quelques exemples illustrant la structuration de l'énoncé assertif de base en allemand :

Tableau 1. Les champs positionnels principaux de l'allemand

\begin{tabular}{|c|c|c|c|c|}
\hline champ 1 & $\begin{array}{l}\text { borne } \\
\text { gauche }\end{array}$ & champ 2 & borne droite & champ 3 \\
\hline $\begin{array}{l}\text { Die Kinder } \\
\text { [Les enfants }\end{array}$ & $\begin{array}{l}\text { mussten } \\
\text { devaient }\end{array}$ & $\begin{array}{l}\text { neben Latein auch } \\
\text { Englisch } \\
\text { outre le latin aussi } \\
\text { l'anglais }\end{array}$ & $\begin{array}{l}\text { lernen. } \\
\text { apprendre.] }\end{array}$ & \\
\hline $\begin{array}{l}\text { Er } \\
{[\mathrm{Il}}\end{array}$ & $\begin{array}{l}\text { hatte } \\
\text { avait }\end{array}$ & $\begin{array}{l}48 \text { seiner } 49 \\
\text { Kämpfe } \\
48 \text { de ses } 49 \\
\text { combats }\end{array}$ & $\begin{array}{l}\text { gewonnen, } \\
\text { gagné, }\end{array}$ & $\begin{array}{l}\text { davon } 38 \\
\text { durch K.O. } \\
\text { dont } 38 \text { par } \\
\text { K.O.] }\end{array}$ \\
\hline $\begin{array}{l}\text { Das Rätsel } \\
\text { [L'énigme }\end{array}$ & $\begin{array}{l}\text { bleibt } \\
\text { reste }\end{array}$ & $\begin{array}{l}\text { bis heute } \\
\text { à ce jour }\end{array}$ & $\begin{array}{l}\text { ungelöst. } \\
\text { non résolue.] }\end{array}$ & \\
\hline $\begin{array}{l}\text { Allerdings } \\
\text { [Toutefois }\end{array}$ & $\begin{array}{l}\text { ist } \\
\text { est }\end{array}$ & $\begin{array}{l}\text { seine Handschrift } \\
\text { son écriture }\end{array}$ & $\begin{array}{l}\text { ein Problem. } \\
\text { un problème.] }\end{array}$ & \\
\hline $\begin{array}{l}\text { Für die } \\
\text { Beteiligten } \\
\text { [Pour les } \\
\text { participants }\end{array}$ & $\begin{array}{l}\text { war } \\
\text { était }\end{array}$ & $\begin{array}{l}\text { das Ausmaß des } \\
\text { Feuers } \\
\text { l'ampleur du feu }\end{array}$ & $\begin{array}{l}\text { eine } \\
\text { Überraschung. } \\
\text { une surprise.] }\end{array}$ & \\
\hline
\end{tabular}

\footnotetext{
${ }^{6}$ L'ordre des compléments dépend par exemple de leur nature syntaxique et de leur cas ; l'ordre des suppléments dépend de leur sémantisme; l'ordre général est soumis à l'intention globale du message.
} 


\section{S. ADAM, C. DeletTres}

Contrairement à ce qui se passe en français, l'organisation des constituants est régressive - elle s'effectue à partir de la borne droite, et en remontant de droite à gauche, l'élément le plus à droite du champ médian (et donc le plus près de la borne droite) étant celui qui est le plus pertinent du point de vue informatif.

(5) Heute will Gottfried allein mit dem Bus nach Hause fahren. nach Hause fahren mit dem Bus nach Hause fahren allein mit dem Bus nach Hause fahren

Cette brève description, si grossière soit-elle, des principes d'organisation de l'énoncé allemand fait malgré tout déjà ressortir l'importance des facteurs (con)textuels et pragmatiques dans l'agencement des constituants. Syntaxiquement parlant l'énoncé allemand se caractérise donc par une grande flexibilité (la seule contrainte syntaxique étant V2). Pour autant, cela ne signifie pas que l'ordre des constituants est libre - il est au contraire largement contraint par des impératifs d'adéquation contextuelle et de cohérence textuelle, et fortement conditionné par l'objectif informatif, communicationnel du locuteur. On verra que ce sont des impératifs de ce type qui expliquent la récurrence des éléments attributifs en tête d'énoncé - malgré leur caractère (syntaxiquement) 'marqué'. Comme le montrent les exemples du tableau précédent, les constituants en fonction attributive représentent généralement des candidats privilégiés à la fonction de borne droite - compte tenu de l'étroit rapport syntaxique qu'ils entretiennent avec le verbe copule, ainsi que de leur valeur prédicative (procédant à la qualification / catégorisation du sujet, ils véhiculent potentiellement l'information essentielle de l'énoncé). Leur projection en tête d'énoncé peut donc être considérée comme marquée - non pas en tant qu'elle s'accompagnerait d'une 'mise en relief' particulière de ces éléments, mais en tant qu'elle va à l'encontre de ce que la structure de base laisserait théoriquement attendre. Pour autant, cette dimension 'marquée' ne porte en aucun cas atteinte à la grammaticalité des énoncés concernés et ne représente pas un marquage stylistique - ces énoncés relèvent du registre standard et se rencontrent couramment dans notre corpus : 
Les EI attributifs allemands et leurs équivalents en français

(6) Ein Beispiel sind die sauren Seen in Skandinavien? ${ }^{7}$ [Un exemple sont les lacs acides en Scandinavie.]

(7) Typische Maßnahmen sind Workshops, Projektgruppen und Lernstätten, an denen meist Führungs- bzw. Nachwuchsführungskräfte teilnehmen ${ }^{8}$.

[Des mesures typiques sont des workshops, des projets de groupe et...]

(8) Nachteilig ist, dass dem Kunden bei Problemen kein direkter Ansprechpartner zur Verfügung steht.

[Préjudiciable est qu'aucun interlocuteur direct ne se tient à la disposition du client en cas de problèmes.]

\subsection{Typologie des EI prédicatifs en allemand}

Les groupes en fonction d'attribut que l'on rencontre en tête d'énoncé sont de trois types : il s'agit de groupes adjectivaux, de groupes nominaux à article zéro, de groupes nominaux avec article (indéfini ou défini).

Le premier type est illustré par les exemples suivants :

(9) Besonders interessant ist das Buch von Ivan Stopar, der in mehreren Bänden alle Burgen und Schlösser Sloweniens beschrieben hat.

[Particulièrement intéressant est le livre de Ivan Stopar, qui a décrit en plusieurs tomes tous les châteaux et châteaux-forts de Slovénie.]

(10) Ärgerlich ist vor allem die Leichtfertigkeit, mit der teils ernsthafte Probleme aufgegriffen und in spekulativer Weise dargestellt werden.

[Embêtant est surtout la légèreté avec laquelle des problèmes en partie sérieux sont traités et présentés spéculativement.]

Ce cas de figure se caractérise non seulement par une fréquence importante ${ }^{9}$, mais également par une grande productivité - on observe en effet pour cette catégorie une grande variété lexicale des adjectifs. Pour des questions de place nous ne listons ici que

\footnotetext{
${ }^{7} 142$ occurrences de Ein Beispiel sind... [Un exemple sont...].

${ }^{8} 122$ occurrences de ADJ + Maßnahmen sind ... [ADJ + mesures sont ...].

${ }^{9}$ Sur l'ensemble du corpus Wikipedia allemand, on relève 5075 structures du type Attribut $_{[\mathrm{AD}]}+$ verbe copule + sujet.
} 


\section{S. AdAm, C. Delettres}

les plus fréquents (nous avons arbitrairement fixé la barre à 10 occurrences) :

auffallend [frappant] (199), auffällig [frappant] (394), außergewöhnlich [exceptionnel] (20), beachtenswert [remarquable] (37), beachtlich [considérable] (13), bedeutsam [significatif] (12), bekannt [connu] (335), belegt [prouvé] (44), bemerkenswert [digne d'attention] (874), bezeichnend [typique] (48), charakteristisch [caractéristique] (43), denkbar [concevable] (86), einzigartig [unique en son genre] (12), entscheidend [décisif] (116), erforderlich [necessaire] (12), erkennbar [visible] (13), erstaunlich [étonnant] (38), erwähnenswert [digne d'être mentionné] (263), gemeinsam [en commun] (34), gewiss [sûr] (10), interessant [intéressant] (290), kennzeichnend [caractéristique] (31), klar [évident] (60), möglich [possible] (175), nachteilig [préjudiciable] (180), neu [nouveau] (56), problematisch [problématique] (130), richtig [juste] (59), sicher [certain] (636), typisch [typique] (38), überliefert [transmis] (18), üblich [courant] (17), unbestritten [incontesté] (38), ungewöhnlich [inhabituel] (88), unstrittig [incontestable] (65), unumstritten [incontesté] (15), unzweifelhaft [indubitable] (11), vorteilhaft [avantageux] (44), wahr [véritable] (16), wahrscheinlich [probable] (37), wesentlich [essentiel] (59), wichtig [important] (401).

Malgré la variété lexicale des attributs adjectivaux topicalisés, il est néanmoins possible d'effectuer certains regroupements sémantiques entre les adjectifs apparaissant dans ces énoncés. Ainsi les adjectifs attributs en tête d'énoncé servent-ils souvent à caractériser l'importance (wichtig / important, entscheidend / décisif), la pertinence (auffallend/remarquable, interessant/ intéressant), le degré de réalité (sicher / certain, wahrscheinlich / probable), la nouveauté (neu/nouveau), l'exactitude (richtig/ correct) du référent encodé par le groupe en fonction sujet. Le fait que les adjectifs relevant de ces notions soient particulièrement bien représentés n'est pas le fruit du hasard mais est à mettre en relation avec la fonction textuelle de la structure - comme nous nous efforcerons de le démontrer au point 1.3.

Parmi les adjectifs rencontrés dans cette position, certains se distinguent par une surreprésentation qui autorise à envisager leur emploi dans cette structure comme relevant de la routine de formulation, c'est-à-dire d'une forme de figement. 
C'est notamment le cas pour les adjectifs bemerkenswert / frappant, sicher / certain, wichtig / important et auffällig / remarquable, qui représentent chacun plus de $10 \%$ des attributs adjectivaux initiaux - et donc quasiment $50 \%$ à eux quatre.

Outre les adjectifs, on retrouve en fonction d'attribut en position initiale des groupes nominaux à article zéro, tels que les énoncés proposés dans les exemples (11) à (13) :

(11) Grund dafür war der Dreißigjährige Krieg. [Raison pour cela était la Guerre de Trente Ans.]

(12) Ursache war ein Bedienfehler der Piloten. [Cause était une fausse manipulation des pilotes.]

(13) Voraussetzung hierfür ist u.A. ein internationaler Zulassungsschein.

[Condition pour cela est entre autres un certificat d'immatriculation international.]

Du point de vue de leur sémantique, la plupart des substantifs apparaissant de façon significativement récurrente à cette position et dans cette fonction ont pour point commun de relever du vocabulaire de l'argumentation; la qualification du groupe sujet qui s'opère par leur intermédiaire consiste à conférer au référent visé par ce sujet le statut d'explication (Grund / raison, Ursache / cause) ou de justification (Ziel / objectif), d'illustration (Beispiel / exemple), de caractérisation (Besonderheit / particularité, Merkmal / trait distinctif, Voraussetzung / préalable, Problem / problème). Ce sont peut-être ces 'restrictions' sémantiques ${ }^{10}$ qui expliquent la moins grande variété lexicale de ce deuxième type par rapport au premier. Comme pour les attributs adjectivaux, les particularités sémantiques des attributs nominaux sont ici aussi en lien direct avec la fonction textuelle des énoncés concernés (voir 1.3).

Du point de vue fonctionnel, le statut des groupes nominaux à article zéro n'est aucunement ambigu : utilisés sans article, ces groupes ne sont pas en emploi référentiel et ne sauraient par conséquent être confondus avec des sujets; il ne

\footnotetext{
${ }^{10} \mathrm{Si}$ on peut parler ici de 'restrictions', il s'agit de restrictions de fait, et non de droit - car a priori bon nombre d'autres substantifs pourraient apparaître sous cette forme et dans cette fonction.
} 


\section{S. AdAm, C. Delettres}

peut s'agir que d'attributs du sujet. Il n'en va pas de même pour le troisième type d'attributs initiaux - à savoir les groupes nominaux avec article (défini ou indéfini), qui en raison de leur forme se distinguent moins facilement des groupes nominaux référentiels en fonction de sujet, comme l'illustrent les exemples (14) et (15), où le groupe Ein Beispiel est tantôt sujet (14), tantôt attribut (15) :

(14) Ein berühmtes Beispiel findet sich in Psycho.

[Un exemple célèbre se trouve dans Psychose.]

(15) Ein Beispiel sind die auch heute noch im Gebrauch befindlichen Symbole der Zodiakzeichen.

[Un exemple sont les symboles des signes du zodiaque toujours en usage aujourd'hui.]

Cette ambiguïté potentielle est néanmoins rarement source de difficulté d'interprétation en contexte - dans la très grande majorité des cas, des indices d'ordre syntaxique ou textuel permettent de clarifier la fonction des groupes. Ainsi dans l'exemple (15) mentionné ci-dessus n'est-il pas possible de considérer le groupe initial Ein Beispiel comme le sujet de l'énoncé, dans la mesure où il n'y a pas concordance formelle avec le verbe ; celui-ci, au pluriel, ne peut avoir pour sujet que le groupe qui le suit immédiatement (die Symbole/les symboles). Quant aux indices d'ordre textuel, ils sont liés au rôle que joue l'énoncé concerné dans la progression/la cohérence textuelle ; c'est sur cet aspect que le point suivant se propose de revenir.

\subsection{Fonctions textuelles}

La grande flexibilité syntaxique qui caractérise l'énoncé assertif allemand et qu'expliquent facteurs exposés au point 1.1 fait que la place des constituants, qui n'est que peu contrainte syntaxiquement, peut être exploitée facilement à des fins pragmatiques - et plus précisément pour assurer une répartition contextuellement adéquate des composantes informatives de l'énoncé ainsi que pour optimiser la cohérence textuelle. Ainsi le champ I estil par exemple prototypiquement le lieu où sont placées les unités qui, indépendamment de leur fonction grammaticale dans l'énoncé, assurent sémantiquement / textuellement l'enchaînement thématique avec le cotexte amont. Symétriquement, le 
(dé)placement d'un constituant à l'extrémité droite du champ médian contribue à lui faire porter le focus informationnel - et le constituant sera d'autant plus focalisé qu'il sera a priori moins 'attendu' à cette position - le point de référence par rapport auquel se définissent les 'attentes' en question étant la structure régressive telle qu'elle a été brièvement définie plus haut.

Les structures sur lesquelles se penche cette contribution constituent une forme de déplacement / d'anticipation en tête d'énoncé d'un élément qui dans l'énoncé de base occupe a priori une position dans la partie droite du champ médian, voire la position de borne droite ; ce procédé relève d'une opération dite de topicalisation - au sens au sens de Bresson (1981, 1993), Samson $(1989,2004)$ ou Dalmas $(2007,2010)$. Les groupes topicalisés sont parfois des éléments thématiques, mais il s'agit le plus souvent d'éléments dont le statut informationnel est rhématique (comme c'est le cas pour les attributs adjectivaux ou nominaux) ; la projection de ces groupes en tête d'énoncé répond à des visées communicatives spécifiques. Les travaux consacrés à ce procédé topologique lui reconnaissent généralement trois fonctions principales :

- l'optimisation de la progression/cohérence textuelle par mise en évidence des enchaînements thématiques; l'élément topicalisé est alors celui qui, dans l'énoncé, entretient le lien le plus direct avec le cotexte amont - soit qu'il constitue un élément explicitement repris de ce cotexte, par reprise littérale ou contigüité sémanticothématique, soit qu'il soit sans difficulté inférable de ce cotexte amont ;

- l'optimisation de la répartition du poids informatif; l'élément portant le poids informatif le plus important étant a priori un élément du champ médian, et même celui situé le plus à droite dans ce champ, il peut être intéressant, pour faciliter le traitement cognitif de l'information par le destinataire (et assurer par là même la plus grande efficacité de la communication), d'évacuer de ce champ médian des unités moins pertinentes du point de vue informatif - car thématiquement accessibles ou déjà connues - pour ne laisser là que 


\section{S. AdAm, C. Delettres}

l'unité / les unités constituant le cœur informatif du message ;

- le marquage de la structure du texte; l'accumulation d'éléments topicalisés relevant de la même thématique peut être exploitée à des fins argumentatives pour mettre en évidence le passage d'un sous-thème à l'autre tout en soulignant la parenté/la continuité thématique.

Dans le cas de la topicalisation de constituants en fonction attributive, la fonction dominante relève de la gestion du poids informatif relatif des composantes informationnelles : il s'agit dans tous les cas de retarder l'apparition du sujet, qui est dans ce cas rhématique et qui constitue le cœur informatif de l'énoncé ; l'évacuation de l'attribut en tête d'énoncé permet de placer la composante la plus informative à l'extrémité droite du champ médian et s'accompagne d'une mise en focus du sujet (cf. 1.1). La fréquence de cette opération s'explique par le fait qu'elle permet de respecter une troisième tendance générale guidant la linéarisation des énoncés - tendance qui consiste à donner l'information ancienne (ou inférable) avant l'information (textuellement) nouvelle ('given before new'). La séquence (16) illustre ce principe :

(16) Der E1 wurde von BMW nie in Serie gefertigt und es existierte auch nur ein Modell dieses Typs. Ein Grund dafür war eine zu geringe Lebensdauer der Batterien.

[La E1 n'a jamais été produite en série par BMW et il n'exista qu'un seul modèle de ce type. Une raison à cela était la durée de vie trop faible des batteries.]

$\mathrm{Au}$ terme de cette première partie, il apparaît que la relativement grande flexibilité syntaxique de l'énoncé assertif allemand offre une grande marge de manœuvre à l'exploitation de la linéarisation des constituants à des fins d'optimisation de la structure informationnelle. Le déplacement / l'anticipation d'une composante relativement peu informative (parce que donnée ou aisément inférable du contexte) en tête d'énoncé permet notamment d'assurer concomitamment le lien thématique avec le cotexte amont et de retarder l'apparition de la composante la plus informative, facilitant et orientant ainsi le travail interprétatif du destinataire et, partant, l'efficacité communicative de l'énoncé. 
Les EI attributifs allemands et leurs équivalents en français

La question qui se pose à ce stade est de savoir quelles stratégies le français, compte tenu des contraintes syntaxiques qui sont les siennes, permet de mettre en œuvre pour s'acquitter d'objectifs communicatifs / textuels similaires.

\section{Contraintes syntaxiques et objectifs textuels - réflexions contrastives}

A la différence de l'énoncé assertif allemand, l'énoncé assertif français se caractérise par une assez forte rigidité syntaxique pour ce qui est de l'ordre des compléments verbaux; en l'absence d'un système de cas, le marquage de la fonction syntaxique des constituants est largement pris en charge par la linéarisation : la fonction d'un constituant est dans une large mesure définie par sa position par rapport au verbe conjugué ${ }^{11}$ :

La vieille dame salue le facteur.

$\mathrm{S} \quad \mathrm{O}$

Le facteur salue la vieille dame ${ }^{12}$.

$\mathrm{S}$

$\mathrm{O}$

Du fait de ces contraintes positionnelles, le français semble a priori moins à même que l'allemand d'exploiter la position relative des constituants pour remplir ses objectifs communicationnels ; en particulier, la simple topicalisation d'un constituant est particulièrement délicate, puisque, consistant à extraire ce

${ }^{11}$ C'est vrai pour les compléments verbaux, c'est en partie vrai également pour les suppléments : la fonction cadrative d'un groupe adverbial est liée à sa position en tête d'énoncé ; placé ailleurs, ce groupe adverbial est intraprédicationnel (Wilmet, $2007: 573$ ) :

(a) A cinq heures, le train est parti $=>$ extraprédicationnel ; cadratif l'énoncé apporte une information sur ce qui s'est passé à une heure précise ;

(b) Le train est parti à cinq heures $=>$ intraprédicationnel : l'énoncé apporte une information sur l'heure à laquelle le train est parti.

Cette distinction entre les objectifs informatifs des deux énoncés est exclusivement liée à la position du groupe adverbial dans l'énoncé.

12 Là où en allemand on pourrait avoir dans le champ I le complément à l'accusatif sans que cela prête à confusion, le cas respectif des groupes permettant d'identifier leur fonction :

Den Briefträger grüßt die alte Dame $=>$ Briefträger : accusatif $>$ objet Die alte Dame grüßt den Briefträger $=>$ Briefträger $:$ nominatif $>$ sujet 


\section{S. AdAm, C. Delettres}

constituant de la position qu'il occupe prototypiquement dans l'énoncé de base, ce procédé topologique risque par définition d'en annuler le marquage fonctionnel. On peut certes trouver quelques (rares) exceptions à ce principe :

(17) Nombreux sont les amateurs de vin d'Alsace à n'avoir jamais entendu parler de ce cépage

(18) Rares sont les ateliers qui ont maintenu leur activité durant cette période.

Il s'agit bien ici de tournures dans lesquelles un adjectif ${ }^{13}$ attribut du sujet est antéposé au verbe. Mais ces cas sont isolés et lexicalisés, figés ; la structure n'est en aucun cas productive et le remplissage lexical n'est absolument pas libre ${ }^{14}$. Et si ces exceptions figées existent avec des attributs adjectivaux, il ne s'en trouve aucun exemple avec des attributs nominaux en tête d'énoncé. Le caractère exceptionnel de l'antéposition de l'attribut n'invalide en aucun cas le constat de la rigidité syntaxique à laquelle sont nécessairement soumis les groupes compléments $\mathrm{du}$ français - et de la faible marge de manœuvre syntaxique dont dispose cette langue en matière de linéarisation des compléments.

Cela étant, les objectifs communicationnels que poursuivent les locuteurs sont les mêmes indépendamment de la langue particulière qu'ils utilisent, et les principes généraux présidant à la gestion de la structure informationnelle, parce qu'ils reflètent une réalité cognitive, sont valables quelle que soit la langue pratiquée ${ }^{15}$. Les locuteurs se trouvent donc placés devant

${ }^{13}$ Il est intéressant de noter ces adjectifs français ne correspondent justement pas aux adjectifs fréquemment topicalisés en allemand - ce qui confirme que le statut de cette structure est différent dans les deux langues.

${ }^{14}$ On relève dans le corpus Wikipédia français 605 cas d'adjectifs attributs en tête d'énoncé ; sur ces 605 cas, $66 \%$ sont des occurrences de 'nombreux/ nombreuses' (400 cas) et $29 \%$ des occurrences de 'rares' ( 175 cas).

${ }^{15}$ Il serait évidemment trop simpliste de prétendre séparer radicalement les principes généraux d'organisation du texte et le système linguistique particulier auquel ont recours les locuteurs; il va de soi que les locuteurs d'une communauté linguistique partagent des préférences de réalisation et des routines de formulation qui conditionnent partiellement leur réception d'un texte et induisent symétriquement des stratégies d'encodage. Mais mutatis mutandis, les principes généraux dont il est question ici - tel que le given-before-new-principle - sont valables supralinguistiquement. 
les mêmes problématiques thématiques et textuelles et doivent y trouver des solutions qui, quant à elles, exploitent les possibilités offertes par chaque système linguistique et qui, surtout, s'accommodent des limites, contraintes et restrictions imposées par ce système.

C'est ce qui explique qu'on retrouve en français, en tête d'énoncé, des éléments sémantiquement équivalents aux attributs adjectivaux et nominaux topicalisés de l'allemand. On a vu plus haut que la fréquence d'occurrence de ces unités en tête d'énoncé s'expliquait par leur rôle dans la progression thématique et argumentative du texte ainsi que par leur caractère de moindre informativité (en comparaison de la composante informative dont leur topicalisation permet de retarder l'apparition). Les équivalents sémantiques français des attributs topicalisés de l'allemand présentent les mêmes caractéristiques informationnelles que ces derniers et jouent un rôle textuel similaire. C'est pourquoi on les retrouve également en tête d'énoncé. Néanmoins vont être développées en français des stratégies syntaxiques différentes de celles mises en œuvre en allemand, de façon à être en adéquation avec les contraintes liées au système linguistique. C'est à la présentation et à l'illustration des plus importantes de ces stratégies qu'est consacrée la prochaine section.

\subsection{Choix du verbe}

D'un point de vue purement syntaxique, on l'a dit, il est difficilement envisageable d'avoir devant le verbe conjugué d'un énoncé non marqué un complément autre que le constituant en fonction de sujet. C'est donc souvent dans cette fonction syntaxique qu'apparaîtront les équivalents français aux attributs nominaux topicalisés de l'allemand.

De ce fait, une des stratégies permettant de concilier la contrainte syntactico-positionnelle et l'objectif communicationnel de retardement de la composante informative essentielle pourra consister à recourir à un verbe d'existence (19), d'inclusion (20) ou de caractérisation (21) :

(19) La majorité des Canadiens qui ont combattu durant la guerre civile américaine l'ont fait dans le camp du Nord. Peu d'entre eux le firent dans le camp confédéré. Un exemple existe cependant, George Ellsworth. 


\section{S. ADAM, C. DELETTRES}

(20) Il apparaît très difficile d'estimer le réel potentiel des personnes manifestant un QI très élevé (ou très bas, dans une moindre mesure). La principale raison réside dans la faiblesse de l'échantillon disponible à ce niveau.

(21) [Le kimono] est formé de rectangles de tissus pliés et cousus, mais jamais recoupés; il est rectiligne, tombant jusqu'aux pieds ou chevilles, suivant la formalité de l'ensemble et la personne qui le porte. Sa particularité consiste dans ses manches très longues, pouvant aller jusqu'au sol pour les kimonos des jeunes femmes.

Dans la séquence (19), le groupe un exemple, indéfini, reprend l'idée de 'nombre peu important' exprimée par le sujet de l'énoncé précédent (Peu d'entre eux), qui appelle une exemplification - celle-ci est proposée en fin d'énoncé sous la forme d'un groupe en apposition (George Ellsworth), syntaxiquement en lien avec le sujet de l'énoncé et correspondant à un remplissage sémantique de ce dernier.

Dans la séquence (20), le sujet du second énoncé (la principale raison) répond directement à une interrogation implicite soulevée par l'énoncé précédent, dans lequel est thématisée la difficulté que représente l'évaluation du QI des individus surdoués - le jugement de valeur contenu dans la tournure impersonnelle il est difficile appelle à être justifié, et le groupe sujet de l'énoncé suivant annonce la réponse à cette interrogation. Le verbe résider dans permet ensuite d'introduire, dans la dernière partie de l'énoncé, le contenu effectif de cette explication - cœur informatif de cet énoncé.

La séquence (21) fonctionne sur un modèle un peu différent - l'ensemble est consacré à la description d'un vêtement traditionnel dont sont présentées les différentes caractéristiques. Le dernier énoncé du passage entend souligner un trait spécifique et distinctif; cette qualification est effectuée par le biais du groupe sujet (sa particularité), dont le lien thématique avec le contexte amont est marqué par la présence du possessif $s a$; la teneur de cette particularité est donnée sous la forme de l'objet prépositionnel du verbe consister.

Ces premières séquences montrent que, même si la structure syntaxique de l'allemand et du français diffèrent dans les exemples de ce type (on rencontrerait en allemand vraisem- 
Les EI attributifs allemands et leurs équivalents en français

blablement des attributs topicalisés en lieu et place des groupes sujets des exemples susmentionnés), c'est à une répartition similaire de l'information qu'on a affaire dans les deux langues - le constituant qui fait le lien (par prolongement, sousthématisation, contraste etc.) avec le contexte amont est placé en tête d'énoncé, tandis que le constituant représentant le cœur informatif de l'énoncé n'apparaît que vers la fin de ce dernier. La structure attributive allemande établit un lien d'équivalence entre l'élément thématique (par le biais du verbe copule sein/ être) ; cette équivalence est moins nette en français compte tenu de la rection des verbes choisis, mais le statut informationnel du constituant placé vers la droite est le même que celui du sujet rhématique de l'allemand.

Le recours à des formes verbales autres que les copules est donc l'une des stratégies sur lesquelles le français peut s'appuyer pour remplir, au moyen d'éléments sémantiquement équivalents, les mêmes objectifs communicationnels que ceux que remplissent en allemand les attributs topicalisés. Cette première tendance présente la particularité (et peut-être l'avantage) de constituer une forme syntaxiquement non marquée - contrairement aux autres stratégies (que nous développerons en 2.2 et 2.3) qui le sont à degrés divers.

\subsection{Diathèse passive}

Une seconde stratégie permettant de placer en position initiale la composante informative 'faible' pour retarder l'apparition de la composante informative principale consiste à exploiter les ressources de la diathèse passive.

(22) La doctrie a été formalisée sur le fait qu'en 1883 le Mexique avait dénoncé la dette contractée par l'empereur Maximilien en promulguant une loi dite «de règlement de la dette nationale » [...]. Le second exemple est fourni par les EtatsUnis, qui ont refusé que Cuba paye les dettes contractée par le régime colonial espagnol.

(23) Les mesures de sécurité lors des célébrations sont complexes, impliquant le Secret Service, mais aussi d'autres agences américaines de sécurité, les cinq branches de l'armée, la police du Capitole et la police métropolitaine du district de Columbia. Une difficulté supplémentaire est posée par la 


\section{S. AdAm, C. Delettres}

possibilité pour les citoyens d'exercer leur droit constitutionnel de manifester, présentant un risque supplémentaire pour les officiels.

(24) Le maître imprimeur, éditeur, de cette fin de XVIII ${ }^{\mathrm{e}}$, début de $\mathrm{XIX}^{\mathrm{e}}$ siècle, devait, tout à la fois, être érudit, entrepreneur, maître artisan et homme du monde. L'érudition était nécessaire à la connaissance des lettres, de l'histoire, du formidable bouillonnement des idées, de la langue française et des langues étrangères. Un exemple nous en est donné dans l'avis de l'éditeur Xhrouet dans le supplément aux lettres de Voltaire. Il y expose son minutieux travail de datation de la correspondance à travers les évènements soient politiques, soient littéraires, du siècle auquel Voltaire faisait allusion dans ses milliers de lettres.

Du point de vue informationnel, les séquences (22) à (24) présentent une structure similaire à celle des énoncés allemands à attribut topicalisé : à l'initiale de l'énoncé concerné, le sujet (groupe en italique) établit le lien thématique avec ce qui précède, tandis que l'information essentielle de l'énoncé est fournie par le complément d'agent (22-23) ou par une indication spatiale (24), en tous les cas par des groupes localisés vers l'aval de l'énoncé.

Un bref commentaire de la séquence (23) illustrera à titre d'exemple le fonctionnement informationnel des séquences de ce type, afin de montrer qu'elles remplissent les mêmes objectifs communicatifs que leurs équivalents allemands à attribut topicalisé. Dans la séquence (23), il est question des enjeux de la sécurité nationale américaine lors de manifestations publiques. D'emblée, la séquence souligne le caractère difficile de la tâche (les mesures... sont complexes), et apporte une première explication à ce jugement : la sécurité est aux mains de multiples organismes (ce qui ne facilite assurément pas la coordination). Le sujet du passage qui nous intéresse contribue à faire progresser le texte en ce sens qu'il introduit un deuxième sous-thème (difficulté supplémentaire) en lien avec la thématique générale (complexité de la gestion de la sécurité nationale); dans le même temps, l'utilisation d'une forme passive permet de retarder l'introduction, sous la forme d'un complément d'agent, de 
Les EI attributifs allemands et leurs équivalents en français

l'élément informatif qui concrétise l'annonce contenue dans le sujet (le droit de manifester).

\subsection{Dislocations à gauche}

Si la topicalisation d'un complément verbal telle que la pratique l'allemand n'est a priori pas possible en français, cela ne signifie pas pour autant que le français n'a pas la possibilité de sortir un complément de la structure de base pour le projeter en tête d'énoncé. Néanmoins, ce déplacement impose généralement le recours à une structure marquée par rapport à la structure de base et par conséquent soumise à des conditions d'emploi spécifiques (en termes contextuels, stylistiques ou plus généralement pragmatiques). Dans le cas qui nous intéresse, la structure marquée qui entre en ligne de compte est la dislocation à gauche. Elle permet la récupération du marquage fonctionnel du constituant disloqué par le biais d'un élément pronominal. L'énoncé (25) illustre ce procédé :

(25) Lui, je ne le crois pas.

Dans cet exemple, l'élément disloqué est le complément d'objet direct, dont la fonction syntaxique est marquée par la reprise pronominale le. Dans le corpus qui sert de base à notre étude, on rencontre des exemples relevant de la dislocation et permettant une gestion de la structure informationnelle comparable - mais pas identique - à celle des énoncés allemands à attribut topicalisé ; il s'agit cependant de cas particuliers de dislocation. Le premier de ces cas est illustré par les exemples (26) à (28) :

(26) Le but, c'est d'obtenir l'abstention du parti socialiste, à l'opposition depuis 1947.

(27) L'idée, c'est d'avoir un exutoire.

(28) L'important, c'est une respiration adéquate.

Le statut particulier de ces exemples tient d'une part au fait que l'élément disloqué est un groupe nominal défini, dont le noyau est un nom abstrait (26-27) ou un adjectif substantivé (28) et qui est repris par le pronom indistinct ${ }^{16} \mathrm{ce} / \mathrm{c}^{\prime}$ (et non par un pronom

${ }^{16}$ Le terme de pronom indistinct est celui proposé par Corblin (1995), relayé par Wilmet (2007). 


\section{S. AdAm, C. Delettres}

personnel comme dans l'exemple (25)). D'autre part, l'élément disloqué occuperait, dans l'énoncé non disloqué correspondant, la fonction de sujet :

(26') Le but est d'obtenir l'abstention du parti socialiste.

(27') L'idée est d'avoir un exutoire.

Ces exemples se caractérisent par une faible fréquence d'occurrence dans le corpus français, qui confirme leur marquage stylistique : il s'agit a priori de structures qui se rencontrent plus fréquemment dans des textes relevant de la conception (et éventuellement de la réalisation) orale ${ }^{17}$.

Un autre type de dislocation est envisageable, qui partage de nombreux points communs avec le type dont il vient d'être question: il s'agit de la structure dite 'pseudo-clivée'. Celle-ci tient sa dénomination de sa parenté formelle avec la structure clivée, puisqu'il est généralement postulé que la structure 'pseudo-clivée' est issue de la combinaison d'une opération de focalisation (par le biais de la clivée) et de topicalisation (par le biais de l'anticipation en tête d'énoncé ${ }^{18}$ ). Anticipant la composante informative la plus faible, ces tournures peuvent en effet être considérées comme des équivalents fonctionnels possibles aux topicalisations d'attributs en allemand. Le fait est cependant que cette structure, stylistiquement marquée comme relevant de l'oral, ne se rencontre que relativement rarement dans les exemples de notre corpus. On trouve certes des cas du type :

(29) Ce qui dérange, c'est le style, il colle au propos.

Mais l'observation du corpus montre que ces exemples sont relativement limités. Le décalage dans la fréquence d'emploi entre les deux langues appelle un commentaire sur la notion d'équivalence. Même si les dislocations à gauche s'apparentent à première vue à la topicalisation allemande en ceci qu'elles

${ }^{17}$ Nous distinguons ici avec Koch \& Österreicher (1985) la réalisation (médiatique) des textes de leur conception.

${ }^{18}$ Pour mémoire, on rappellera que le terme de 'clivée' désigne des énoncés tels que : C'est à Paris qu'il a rencontré sa femme, c'est pour lui que j'ai fait tout cela, etc. 
consistent à projeter en tête d'énoncé un complément autre que le sujet, il convient toutefois de repréciser que dislocation et topicalisation ne sont pas totalement assimilables. A la différence du procédé allemand, le déplacement d'un complément s'accompagne en français obligatoirement du marquage du déplacement (1) par l'adjonction d'une virgule avant la reprise de la structure de base, et surtout (2) par une reprise pronominale qui explicite la fonction syntaxique du constituant déplacé. A ce titre, la dislocation à gauche est syntaxiquement plus marquée que la topicalisation allemande. On relèvera par ailleurs deux autres distinctions : d'une part, la dislocation à gauche est un procédé très fréquemment employé pour redoubler le sujet (cela revient à le thématiser, à souligner son statut thématique), ce qui n'est pas le cas de la topicalisation allemande, qui concerne essentiellement des constituants remplissant d'autres fonctions. Les exemples - non issus du corpus - comme (30) et (31) n'ont pas pour équivalents des topicalisations allemandes :

(30) Pierre, il exagère.

(31) Moi, je trouve qu'il a raison.

Il faut par ailleurs rappeler que la dislocation à gauche est un procédé qui existe également en allemand ${ }^{19}$.

Topicalisation et dislocation sont donc bien des procédés distincts. Il apparaît au final qu'on peut considérer les différentes formes françaises ici illustrées comme autant d'équivalents de la structure allemande décrite sous 1.3 , tout en mettant en lumière la multiplicité des stratégies discursives que les locuteurs peuvent choisir d'appliquer ou pas ; cela étant, il n'en reste pas moins que ces structures, marquées, sont plus usitées dans d'autres genres textuels que ceux dont se compose notre corpus de base - notamment dans des types de discours relevant de l'oral.

Un dernier cas nous semble mériter qu'on s'y attarde, celui de pseudo-clivées, où la position sujet est occupée par un présentatif (Ce qui $X, \ldots$ c'est...), le groupe relatif neutre

${ }^{19}$ Un énoncé tel que celui proposé ci-dessous constituerait un exemple de dislocation à gauche en allemand :

Den Paul, den habe ich schon lange nicht mehr gesehen.

[Le Paul, je ne l'ai pas vu depuis longtemps.] 


\section{S. ADAM, C. Delettres}

anticipé comportant les informations thématiques qui permettent d'établir le lien avec le cotexte amont. L'énoncé (32) illustre le cas auquel nous avons affaire ici :

(32) Ce qui nous manque, c'est un peu de merveille.

Il convient cependant de préciser que ce troisième type, relevant plutôt de l'oral (comme les précédents), est relativement peu représenté dans le corpus Wikipédia français.

\subsection{Forme de l'élément thématique}

L'observation du corpus a permis de relever un dernier cas intéressant du point de vue contrastif. Il s'agit de cas où la fonction sujet à l'initiale est sémantiquement remplie par les éléments qui apparaissent en position d'attributs topicalisés en allemand, mais qui sont en français étoffés de qualifications adjectivales (33) ou intégrés à une structure pronominale (34) :

(33) Une explication possible est la grande distance séparant l'archipel et les principales terres continentales riches en biodiversité.

(34) Un des objectifs de l'expertise médico-légale est de déterminer la part de responsabilité entre l'armée et le malade.

La raison pour laquelle ces tournures sont relativement fréquentes nous semble tenir au fait que, par le biais de l'adjectif ou de la sélection pronominale, se trouve clarifiée la contribution de ces constituants à la progression textuelle (l'adjectif ou la sélection par le biais du pronom indéfini prennent en charge l'enchaînement). En allemand en revanche, ces mêmes unités lexicales sont employées sans modifieur.

Si l'on dresse le bilan des observations faites dans cette deuxième partie, on constate notamment que :

- En français, les unités lexicales en position initiale équivalentes aux unités en fonction d'attribut allemandes sont toujours en position de sujet syntaxique - ce qui est, à quelques rares exceptions lexicalisées près, la seule fonction que peut remplir un GN préverbal dans un énoncé non marqué ; un changement de verbe ou de voix peut dans certains cas permettre de retarder le sujet rhématique porteur de l'information nouvelle. 
- D'autres stratégies courantes (mais pas spécialement dans ce type de corpus) sont le recours aux structures disloquée ou pseudo-clivée; ces structures ne correspondent en effet pas vraiment au type de texte informationnel qui se veut scientifique mais se retrouvent davantage dans des genres textuels relevant de la conception orale.

Si une projection d'éléments en fonction d'attribut n'est pas envisageable telle quelle en français (à part les exceptions évoquées dans les exemples (17) et (18)), compte tenu des contraintes syntaxiques et positionnelles propres à cette langue, la comparaison entreprise dans cette seconde partie nous a permis de constater que l'on peut retrouver malgré tout en grande partie les mêmes contenus sémantiques en position initiale et que ces unités remplissent des fonctions similaires à celles des structures attributives allemandes pour ce qui est de la répartition de l'information au sein de l'énoncé et donc de la gestion de la progression textuelle, mais qu'elles présentent d'importantes différences avec les structures allemandes - différences en termes de fréquence et surtout en termes de fonction (syntaxique).

\section{Conclusion}

La comparaison entre le français et l'allemand met donc en lumière une situation très intéressante: alors que les éléments initiaux étudiés divergent radicalement quant à leur fonction syntaxique (en allemand, les éléments initiaux étudiés sont clairement des attributs alors qu'en français, leurs 'équivalents' sont forcément des sujets syntaxiques) et donnent lieu à des faits de langue différents (topicalisation en allemand, structure non marquée en français), on s'aperçoit qu'ils sont mis en œuvre dans les deux langues pour remplir des fonctions textuelles similaires: dans les deux cas, ils permettent d'ouvrir l'énoncé sur un élément assurant l'enchaînement avec le contexte amont et de réserver pour la partie droite de l'énoncé - c'est-à-dire la partie portant le focus par défaut - la composante informative essentielle de l'énoncé.

Nous espérons que cette étude sur les éléments initiaux, étayée par des corpus parallèles authentiques, aura contribué à 


\section{S. AdAm, C. Delettres}

montrer la primauté des objectifs communicatifs dans la constitution du texte, puisqu'il apparaît qu'au-delà des différences fonctionnelles liées aux contraintes imposées par un système linguistique donné, on retrouve mises en œuvre par les locuteurs de différentes langues les mêmes stratégies de gestion de la répartition de l'information.

\section{Bibliographie sélective}

Baudot D. (1992). « Gegessen wurde nichts. La mise en position initiale de la base verbale passive : enchaînement phrastique et mise en relief d'une unité dynamique », Nouveaux Cahiers d'allemand 1992/2: 207-214.

Baudot D. (1993). «Informative und kommunikative Funktionen der Erst-, Letzt- und Nachstellung unter besonderer Berücksichtigung von Passivsätzen », in J.-F. Marillier (éd.), Satzanfang - Satzende. Syntaktische, semantische und pragmatische Untersuchungen zur Satzabgrenzung und Extraposition im Deutschen. Tübingen : Narr, 233-248.

Bresson D. (1993). «Prosodie, Syntax und Semantik der Hervorhebung am Satzanfang », in J.-F. Marillier (éd.), Satzanfang - Satzende. Syntaktische, semantische und pragmatische Untersuchungen zur Satzabgrenzung und Extraposition im Deutschen. Tübingen : Narr, 73-84.

Corblin F. (1995). Les formes de reprises dans le discours. Rennes : Presses universitaires de Rennes.

Dalmas M. (2007). «Aspekte der Topikalisierung», in M. Tarvas (éd.), Linguistik und Didaktik. Beiträge der Tagung " Tradition und Zukunft der Germanistik». Seeria Germanistik in Tallinn: Texte, Thesen und Projekte zur deutschen Sprache und Literatur. Tallinn : TLÜ Kirjastus, 83-94.

Dalmas M. (2010). « Topikalisierung als Mittel zur Textstrukturierung ", in I. Bartozsewicz, M. Dalmas, J. Szczęk \& A. Tworek (éd.), Germanistische Linguistik extra muros Aufforderungen. Wroclaw, Dresden : Neisse Verlag, 45-54. 
Les EI attributifs allemands et leurs équivalents en français

Doherty M. (2005). « Topic-worthiness in German and English », Linguistics 43/1. Berlin : De Gryuter, 181-206.

Koch P. \& Österreicher W. (1985). « Sprache der Nähe - Sprache der Distanz. Mündlichkeit und Schriftlichkeit im Spannungsfeld von Sprachtheorie und Sprachgeschichte ", in Romanistisches Jahrbuch 36/85 : 15-43.

Krifka M. (2007). « Basic notions of information structure», in C. Féry, G. Fanselow \& M. Krifka (éd.), Interdisciplinary Studies of Information Structure 6. Potsdam : Universitätsverlag Potsdam, 13-56.

Higginbotham J. (1987). «Indefiniteness and predication », in A. ter Meulen \& E. J. Reuland (éd.) The Representation of (In) definiteness. Cambridge : Mass Press, 43-70.

Kupietz M. \& Keibel H. (2009). « The Mannheim German Reference Corpus (DEREKO) as a basis for empirical linguistic research », in M. Minegishi \& Y. Kawaguchi (éd.), Working Papers in Corpus-based Linguistics and Language Education, No. 3. Tokyo: Tokyo University of Foreign Studies (TUFS), 53-59.

Kupietz M., Belica C., Keibel H. \& Witt A. (2010). " The German Reference Corpus DeREKo : A primordial sample for linguistic research », in N. Calzolari et al. (éd.), Proceedings of the 7th conference on International Language Resources and Evaluation (LREC 2010). Valletta : European Language Resources Association (ELRA), 1848-1854. http://www.lrec-conf.org/proceedings/lrec2010/pdf/414 Paper.pdf.

Roy I. (2009). « Typologie des prédicats non verbaux dans les phrases copulatives et théorie de la prédication », in A.H. Ibrahim (éd.), Prédicats, prédication et structures prédicatives. Paris: Cellule de recherche en linguistique, 131-147.

Samson G. (1989). «Rhematische Erstbesetzung : Kommunikative Ebene und Textkohärenz », in E. Faucher, F. Hartweg \& J. Janitza (éd.), Sens et Etre. Mélanges en l'honneur de J.M. Zemb. Nancy : Presses universitaires de Nancy, 207225. 


\section{S. AdAM, C. Delettres}

Samson G. (2004). «L'attaque de l'énoncé assertif en allemand », in P. Cotte, M. Dalmas \& H. Wlodarczyk (éd.), Enoncer. L'ordre informatif dans les langues. Paris: L'Harmattan, 185-206.

Stark E. (2006). Indefinitheit und Textkohärenz: Entstehung und semantische Strukturierung. Berlin : de Gruyter.

Wilmet M. (2007). Grammaire critique du français. Bruxelles : De Boeck.

Zemb J.-M. (1968). Les structures logiques de la proposition allemande. Paris : O.C.D.L.

Zemb J.-M. (1993). "Remarques sur l'ouverture X de p.», Nouveaux Cahiers d'allemand 93/1 : 2-14. 